\title{
LETTER
}

\section{Clinical predictors of mortality due to COVID-19 based on an analysis of data of 150 patients from Wuhan, China}

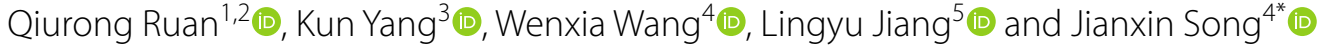

(C) 2020 Springer-Verlag GmbH Germany, part of Springer Nature

Dear Editor,

The rapid emergence of COVID-19 in Wuhan city, Hubei Province, China, has resulted in thousands of deaths [1]. Many infected patients, however, presented mild flu-like symptoms and quickly recover [2]. To effectively prioritize resources for patients with the highest risk, we identified clinical predictors of mild and severe patient outcomes.

Using the database of Jin Yin-tan Hospital and Tongji Hospital, we conducted a retrospective multicenter study of 68 death cases $(68 / 150,45 \%)$ and 82 discharged cases $(82 / 150,55 \%)$ with laboratory-confirmed infection of SARS-CoV-2. Patients met the discharge criteria if they had no fever for at least 3 days, significantly improved respiratory function, and had negative SARS-CoV-2 laboratory test results twice in succession. Case data included demographics, clinical characteristics, laboratory results, treatment options and outcomes. For statistical analysis, we represented continuous measurements as means (SDs) or as medians (IQRs) which compared with Student's $t$ test or the Mann-Whitney-Wilcoxon test. Categorical variables were expressed as numbers (\%) and compared by the $\chi^{2}$ test or Fisher's exact test.

The distribution of the enrolled patients' age is shown in Fig. 1a. There was a significant difference in age between the death group and the discharge group $(p<0.001)$ but no difference in the sex ratio $(p=0.43)$. A total of $63 \%(43 / 68)$ of patients in the death group and

\footnotetext{
*Correspondence: songsingsjx@sina.com

${ }^{4}$ Department of Infectious Diseases, Tongji Hospital, Tongji Medical College, Huazhong University of Science and Technology, 1095 Jiefang Avenue, Wuhan 430030, Hubei, China

Full author information is available at the end of the article
}

Qiurong Ruan and Kun Yang have contributed equally to this work.
$41 \%(34 / 82)$ in the discharge group had underlying diseases $(p=0.0069)$. It should be noted that patients with cardiovascular diseases have a significantly increased risk of death when they are infected with SARS-CoV-2 $(p<0.001)$. A total of $16 \%(11 / 68)$ of the patients in the death group had secondary infections, and $1 \%(1 / 82)$ of the patients in the discharge group had secondary infections $(p=0.0018)$. Laboratory results showed that there were significant differences in white blood cell counts, absolute values of lymphocytes, platelets, albumin, total bilirubin, blood urea nitrogen, blood creatinine, myoglobin, cardiac troponin, C-reactive protein (CRP) and interleukin-6 (IL-6) between the two groups (Fig. 1b and Supplementary Table 1).

The survival times of the enrolled patients in the death group were analyzed. The distribution of survival time from disease onset to death showed two peaks, with the first one at approximately 14 days (22 cases) and the second one at approximately 22 days (17 cases) (Fig. 1c). An analysis of the cause of death was performed. Among the 68 fatal cases, 36 patients (53\%) died of respiratory failure, five patients (7\%) with myocardial damage died of circulatory failure, 22 patients (33\%) died of both, and five remaining died of an unknown cause (Fig. 1d). Based on the analysis of the clinical data, we confirmed that some patients died of fulminant myocarditis. In this study, we first reported that the infection of SARS-CoV-2 may cause fulminant myocarditis. Given that fulminant myocarditis is characterized by a rapid progress and a severe state of illness [3], our results should alert physicians to pay attention not only to the symptoms of respiratory dysfunction but also the symptoms of cardiac injury. 
A
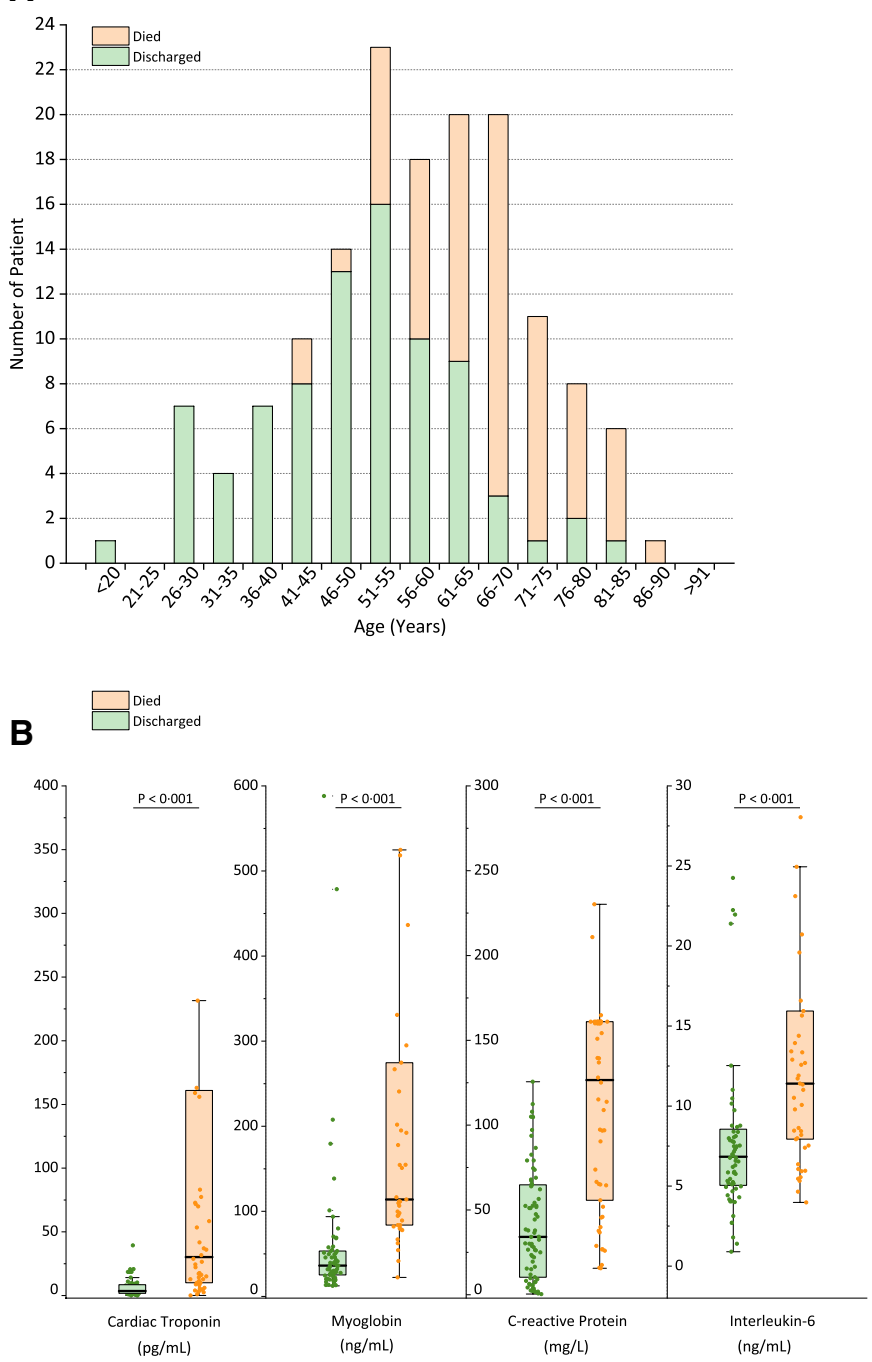

C

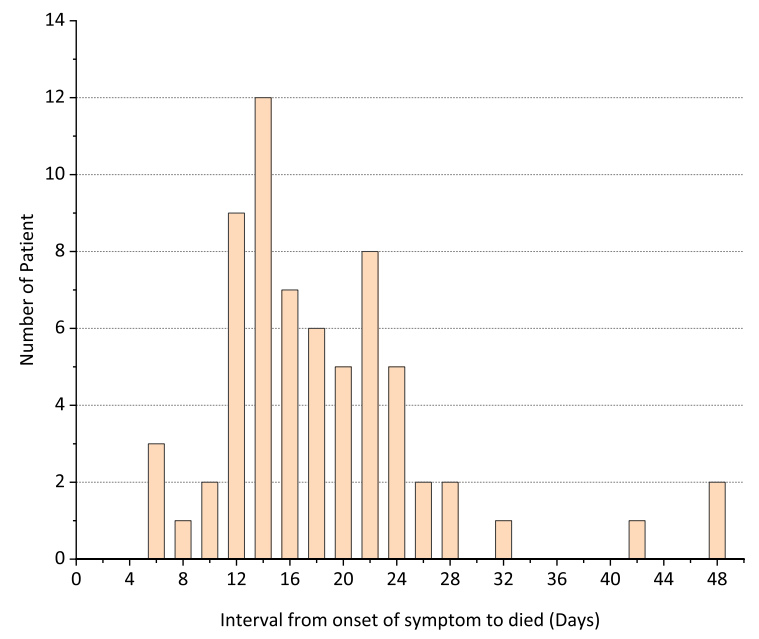

D

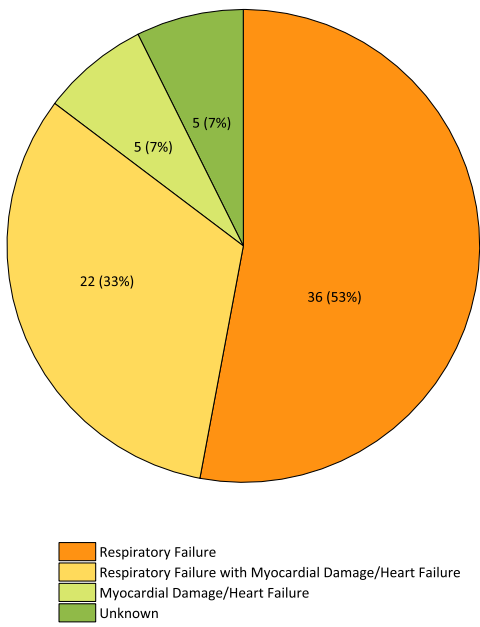

Fig. 1 a Age distribution of patients with confirmed COVID-19; b key laboratory parameters for the outcomes of patients with confirmed COVID19 ; c interval from onset of symptom to death of patients with confirmed COVID-19; d summary of the cause of death of 68 died patients with confirmed COVID-19

Further, large-scale studies and the studies on autopsy are needed to confirm our analysis.

In conclusion, predictors of a fatal outcome in COVID19 cases included age, the presence of underlying diseases, the presence of secondary infection and elevated inflammatory indicators in the blood. The results obtained from this study also suggest that COVID-19 mortality might be due to virus-activated "cytokine storm syndrome" or fulminant myocarditis.

\section{Electronic supplementary material}

The online version of this article (https://doi.org/10.1007/s00134-020-05991-x) contains supplementary material, which is available to authorized users.

\section{Author details}

${ }^{1}$ Institute of Pathology, Tongji Hospital, Tongji Medical College, Huazhong University of Science and Technology, 1095 Jiefang Avenue, Wuhan 430030, Hubei, China. ${ }^{2}$ Department of Pathology, School of Basic Medicine, Tongji Medical College, Huazhong University of Science and Technology, 1095 Jiefang Avenue, Wuhan 430030, Hubei, China. ${ }^{3}$ Department of Dermatology, Tongji Hospital, Tongji Medical College, Huazhong University of Science and Technology, 1095 Jiefang Avenue, Wuhan 430030, Hubei, China. ${ }^{4}$ Department of Infectious Diseases, Tongji Hospital, Tongji Medical College, Huazhong University of Science and Technology, 1095 Jiefang Avenue, Wuhan 430030, Hubei, China. ${ }^{5}$ Department of Clinical Immunology, Tongji Hospital, Tongji Medical College, Huazhong University of Science and Technology, 1095 Jiefang Avenue, Wuhan 430030, Hubei, China.

\section{Acknowledgements}

We are indebted to Prof. Tie Chen and Prof. Daowen Wang, who helped to prepare and review the manuscript. We also thank Drs. Yanping Cai, Yin Lv, Jia Chen, Nana Xie, Jiling Zhu, Mingyou Xing, Yuan Song, Chaolin Huang, Li Zhang 
and Xingjie Hao for collecting cases or scientific support. And we gratefully acknowledge all the health care workers on the front line and all the patients involved in the study.

\section{Authors contribution}

JXS, QRR and KY contributed to the conception and design of the study, had full access to all the data in the study and take responsibility for the integrity of the data and the accuracy of the data analysis. KY, WXW and LYJ contributed to the acquisition of data. KY, WXW, LYJ and JXS contributed to the analysis and interpretation of the data. KY and WXW contributed to the statistical analyses. All authors participated in manuscript writing, revision and approval for final submission. Drs. QRR and KY contributed equally and shared first authorship.

\section{Funding}

This work was supported by the Emergency Project for the Prevention and Control of the Novel Coronavirus (SARS-CoV-2) Outbreak in Hubei Province, China, as well as the National Natural Science Foundation of China (81703174 and 81602722). The funding source had no role in the design and conduct of the study, the analysis and interpretation of the data, or in the writing of the manuscript.

\section{Compliance with ethical standards}

\section{Conflicts of interest}

All authors declare no competing interests.

\section{Ethical approval}

This study was approved by the Ethics Commission of Jin Yin-tan Hospital (KY2020-14.01) and the Ethics Commission of Tongji Hospital (TJ-C20200125).

\section{Publisher's Note}

Springer Nature remains neutral with regard to jurisdictional claims in published maps and institutional affiliations.

Accepted: 24 February 2020

Published online: 3 March 2020

\section{References}

1. Mahase E (2020) Coronavirus: covid-19 has killed more people than SARS and MERS combined, despite lower case fatality rate. BMJ 368:m641. https://doi.org/10.1136/bmj.m641

2. Chen N, Zhou M, Dong X, Qu J, Gong F, Han Y, Qiu Y, Wang J, Liu Y, Wei Y, Xia J, T Yu, Zhang X, Zhang L (2020) Epidemiological and clinical characteristics of 99 cases of 2019 novel coronavirus pneumonia in Wuhan, China: a descriptive study. Lancet. https://doi.org/10.1016/s0140 $-6736(20) 30211-7$

3. Wang D, Li S, Jiang J, Yan J, Zhao C, Wang Y, Ma Y, Zeng H, Guo X, Wang H, Tang J, Zuo H, Lin L, Cui G, Section of Precision Medicine Group of Chinese Society of Cardiology, Editorial Board of Chinese Journal of Cardiology, Working Group of Adult Fulminant Myocarditis (2019) Chinese society of cardiology expert consensus statement on the diagnosis and treatment of adult fulminant myocarditis. Sci China Life Sci 62(2):187-202. https://doi.org/10.1007/s11427-018-9385-3 